\title{
CONCERNING CERTAIN EQUICONTINUOUS SYSTEMS OF CURVES*
}

In order that a system $G$ of open curves lying in a given plane $S$ should be equivalent, from the standpoint of analysis situs, $\dagger$ to a complete $\dagger$ system of parallel lines in $S$ it is not sufficient that through each point of $S$ there should

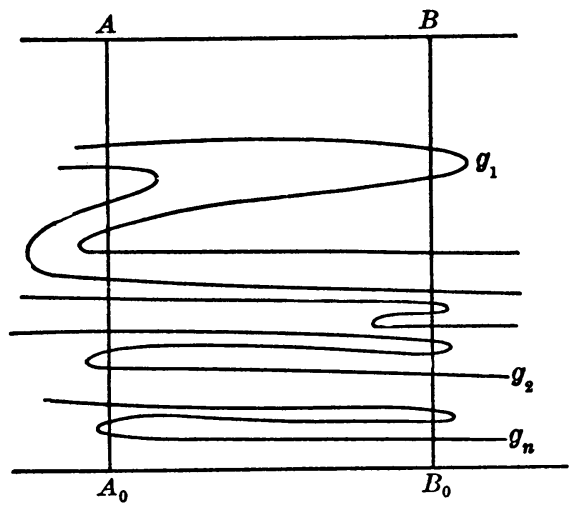

FIG. 1.

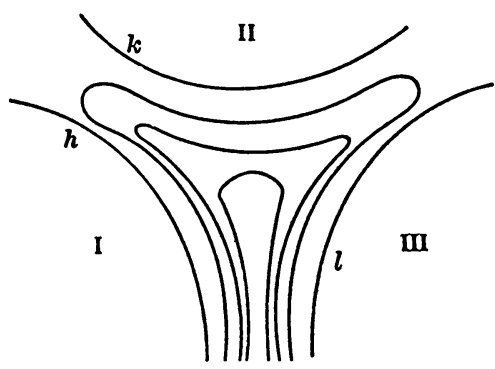

FIG. 2.

pass one and only one curve of the system $G$. Consider the examples indicated in Figs. 1 and $2 . \ddagger$ In each of these examples through each point of the plane there is one and only one curve of the system in question but the system

* Cf. papers presented to the Society, April 28 and October 27, 1917.

† A complete system of parallel lines in a plane $S$ is the set of all lines in $S$ parallel to a given line. A system $G$ of open curves is said to be equivalent, from the standpoint of analysis situs, to such a system of lines $L$ if there is a one to one continuous transformation of $S$ into itself which carries $G$ into $L$.

$\ddagger$ In the case roughly indicated by Fig. $1, A_{0} B_{0}$ and $A B$ are two parallel lines at a distance apart equal to 1 . These lines both belong to the system $G$ and so does every line which is parallel to them but which does not lie between them. For each positive integer $n, g_{n}$ is an open curve belonging to $G$ such that (1) there is an interval of $g_{n}$ that contains a point of $B_{0} B$ but has its endpoints on $A_{0} A$, (2) every point of $g_{n}$ is at a distance of less than $1 / n$ from the line $A_{0} B_{0}$. Of course, as is indicated in Fig. 1 for the case $n=1$, there does not exist, on every curve of $G$ that lies between $g_{n}$ and $g_{n+1}$, an interval that contains a point of $B_{0} B$ and has its endpoints on $A_{0} A$.

In the example indicated in Fig. 2, the open curves $h, k$ and $l$ belong to $G$. To obtain the curves of $G$ which lie in domain I, II or III construct through each point of that domain an open curve parallel and congruent to $h, k$ or $l$ respectively. Each curve of $G$ that lies in the domain bounded by $h, k$ and $l$ lies as is roughly suggested in the figure. 
is not in one to one continuous correspondence with a complete system of parallel lines. Let $G_{1}$ and $G_{2}$ be the system of curves represented in Figs. 1 and 2 respectively. The system $G_{1}$ is not equicontinuous. ${ }^{*}$ That is to say it is not true that for every positive number $\epsilon$ there exists a positive number $\delta$. such that if $P_{1}$ and $P_{2}$ are points on some curve $g$ of $G$ at a distance apart less than $\delta_{e}$ then that arc of $g$ which has $P_{1}$ and $P_{2}$ as its endpoints lies wholly within some circle of radius $\epsilon$. The system $G_{2}$ is equicontinuous but fails to be what I will call inversely equicontinuous.

Definition 1. A system of curves $G$ is equicontinuous with respect to a given point-set $M$ if for every positive number $\epsilon$ there exists a positive number $\delta_{M_{e}}$ such that if $P_{1}$ and $P_{2}$ are two points of $M$ at a distance apart less than $\delta_{M_{e}}$ and lying on a curve $g$ of the system $G$ then that arc of $g$ which has $P_{1}$ and $P_{2}$ as endpoints lies wholly within some circle of radius $\epsilon$.

Definition 2. A system of curves $G$ is inversely equicontinuous with respect to a point-set $M$ if for every positive number $\epsilon$ there exists a positive number $\delta_{M e}$ such that if $P_{1}$ and $P_{2}$ are two points of $M$ at a distance apart less than $\epsilon$ and lying on a curve $g$ of the system $G$ then that interval of $g$ which has $P_{1}$ and $P_{2}$ as endpoints lies wholly within a circle of radius $\delta_{M_{e}}$.

I will show that if $G$ is a system of open curves lying in $S$ such that through each point of $S$ there is just one curve of $G$, then in order that the system $G$ should be equivalent, from the standpoint of analysis situs, to a complete system of parallel straight lines it is necessary and sufficient that it should be both equicontinuous and inversely equicontinuous with respect to every bounded set of points. Additional theorems of a related nature will also be established.

Theorem 1. Suppose that, in a given plane $S, A B C D$ is a rectangle and $G$ is a set of arcs such that (1) through each point of the point-set $\vec{R}$, composed of $A B C D$ and its interior $R$, there is just one arc of $G$, (2) $B C$ and $A D$ are arcs of $G$, (3) every arc of $G$ (with the exception of $B C$ and $A D$ ) lies entirely within $A B C D$ except that its endpoints are on $A B$ and $C D$ respectively, (4) the set of arcs $G$ is equicontinuous.

Then there is a one to one continuous transformation of the plane $S$ into itself which transforms the rectangle $A B C D$ into a rectangle $A^{\prime} B^{\prime} C^{\prime} D^{\prime}$ and transforms the set of arcs $G$ into the set of all straight line intervals which are parallel to $A^{\prime} D^{\prime}$ and lie between $A^{\prime} D^{\prime}$ and $B^{\prime} C^{\prime}$ (except that one of them coincides with $A^{\prime} D^{\prime}$ and another with $B^{\prime} C^{\prime}$ ) and are terminated by $A^{\prime} B^{\prime}$ and $C^{\prime} D^{\prime}$.

The truth of this theorem will be established with the help of a lemma. This lemma will be proved first.

Definition 3. A connected domain $K$ is said to be a simple domain with

* Cf. G. Ascoli, Sulle curve limiti di una variétà data di curve, M e m or i e d e 11 a $\mathrm{R}$ e a le A c c a de mia dei Lincei, vol. 18 (1884), pp. 521-586. 
respect to $a$ set of arcs $G$ satisfying the conditions stated in the hypothesis of Theorem 1 if (1) every point of $K$ is within $A B C D$, (2) $K$ contains the whole of every $G$-interval* whose endpoints are in $K$, (3) there exisi two $G$-arcs $g_{1}$ and $g_{2}$ such that $(a) g_{1}$ lies above $g_{2}$, every point of $K$ is between $g_{1}$ and $g_{2}$ and both $g_{1}$ and $g_{2}$ have points in common with the boundary of $K,(b)$ the set of all those points that the boundary of $K$ has in common with $g_{i}$ is an interval $t_{i}$ of $g_{i}(i=1,2),(c)$ no point of $t_{1}$ or of $t_{2}$ is a limit point of a point-set which lies between $g_{1}$ and $g_{2}$ and contains no point of $K$. The interval $t_{i}$ minus its endpoints will be called the upper base, and the interval $t_{2}$ minus its endpoints will be called the lower base, of the domain $K$.

LEMмA 1. If $G$ is a set of arcs satisfying the conditions stated in the hypothesis of Theorem 1 and $K$ is a simple domain with respect to $G$, then any point on the upper base of $K$ can be joined to any point on its lower base by a simple continuous arc that lies wholly in $K$ and does not have more than one point in common with any arc of the set $G$.

Proof. If $P$ is a point of $R$ and $\epsilon$ is a positive number let $R_{P e}$ denote the set of all points $X$ such that $X$ lies on a $G$-interval whose endpoints are both within a circle of radius $\epsilon$ with center at $P$. If for a given point $P$ and a given pair of positive numbers $e$ and $\epsilon$, such that $e \leqq \epsilon$, the point-set $R_{P e}$ has points between two distinct $G$-arcs $g_{1}$ and $g_{2}$ and also has points on $g_{1}$ and points on $g_{2}$, the set of all those points of $R_{P e}$ that lie between $g_{1}$ and $g_{2}$ will be called an elemental region of rank $\epsilon . \dagger$ It may be easily proved that if $\epsilon$ is a positive number each point of $K$ is in some elemental region of rank $\epsilon$ which lies together with its boundary wholly in the point-set $K^{*}$ composed of $K$ and its two bases. Such an elemental region will be called a $K$-element of rank $\epsilon$. If $E$ and $F$ are two points of $K^{*}$ and $E$ is above $F$, a chain of $K$-elements from $E$ to $F$ or from $F$ to $E$ or joining $E$ to $F$ or $F$ to $E$ is a finite set of $K$-elements $K_{1}, K_{2}$, $K_{3}, \cdots, K_{n}$ such that (1) $E$ belongs to the upper base of $K_{1}$ and $F$ belongs to the lower base of $K_{n}$, (2) for each $i(1 \leqq i \leqq n)$ the lower base of $K_{i}$ and the upper base of $K_{i+1}$ lie on the same arc of the set $G$ and have points in common and the set of all their common points is a segment $t_{i}$. The point-set $K_{1}+K_{2}+K_{3}+\cdots+K_{n}+t_{1}+t_{2}+t_{3}+\cdots+t_{n-1}$ is a simple domain. It will be called the domain associated with the chain $K_{1}, K_{2}, \cdots, K_{n}$. Suppose that $E$ is a point on the upper base of $K, F$ is a point on its lower base and $\epsilon$ is a positive number. I will show that $E$ can be joined to $F$ by a chain of $K$-elements of rank $\epsilon$. Let $\bar{K}$ denote the set of all those points of $K$

* If $G$ is a set of arcs or curves a $G$-arc or a $G$-curve is an arc or a curve of the set $G$. A $G$-interval is an interval (and a $G$-segment is a segment) of such an arc or curve. If $G$ is a set satisfying the conditions stated in the hypothesis of Theorem 1 , the $G$-arc $g_{1}$ is said to be abova the $G$-arc $g_{2}$ if it lies between $g_{2}$ and $B C$. If $P$ is a point of $\vec{R}, g_{P}$ denotes that arc of $G$ which contains $P$. If $P_{1}$ and $P_{2}$ are points of $\bar{R}, P_{1}$ will be said to lie above $P_{2}$ in case $g_{P_{1}}$ is above $g_{P_{2}}$.

$\dagger$ According to this definition if $\epsilon_{1}<\epsilon_{2}$ every elemental region of rank $\epsilon_{1}$ is also of rank $\epsilon_{2}$. 
that lie on arcs of $G$ below the arc $g_{E}$ and that can be joined to $E$ by chains of $K$-elements of rank $\epsilon$. There exists a $K$-element of rank $\epsilon$ whose upper base contains the point $E$ and every such $K$-element contains points in common with some $g$-arc lying below $g_{E}$. It follows that the set $\bar{K}$ exists.

Suppose that $W Z$ is an arc of $G$ that contains a point of $\bar{K}$. The set of points common to $W Z$ and $K$ is a segment $W^{\prime} Z^{\prime}$. Every point of $W^{\prime} Z^{\prime}$ must belong to $\bar{K}$. For suppose this is not the case. Then the segment $W^{\prime} Z^{\prime}$ is the sum of two mutually exclusive point-sets $S_{1}$ and $S_{2}$ such that $S_{1}$ is a subset of $\bar{K}$ but no point of $S_{2}$ belongs to $\bar{K}$. There exists a point $P$ which either belongs to $S_{1}$ and is a limit point of $S_{2}$ or belongs to $S_{2}$ and is a limit point of $S_{1}$. In the first case there is a chain $\alpha_{2}$ of $K$-elements of rank $\epsilon$ from $E$ to $P$. The lower base of the last element of this chain is a segment of $W^{\prime} Z^{\prime}$ containing $P$. Since $P$ is a limit point of $S_{2}$ this segment must contain at least one point $P_{2}$ of $S_{2}$. Thus $\alpha_{2}$ is a chain of $K$-elements of rank $\epsilon$ from $E$ to $P_{2}$. Thus the supposition that $S_{1}$ contains a limit point of $S_{2}$ leads to a contradiction. Suppose now that $S_{2}$ contains a point $P$ which is a limit point of $S_{1}$. There exists (Fig. 3) a $K$-element $e$ of rank $\epsilon$ whose lower base

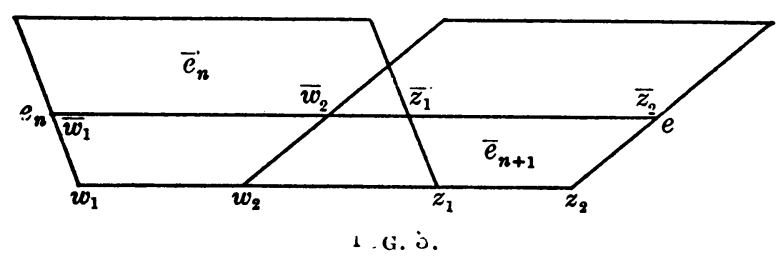

$W_{2} Z_{2}$ is a segment of $W^{\prime} Z^{\prime}$ containing $P$. Since $P$ is a limit point of $S_{1}$ there exists on the segment $W_{2} Z_{2}$ a point $P_{1}$ belonging to $S_{1}$. There exists a chain $e_{1}, e_{2}, e_{3}, \cdots, e_{n}$ of $K$-elements of rank $\epsilon$ from $E$ to $P_{1}$. The lower base of the last element $c_{n}$ of this chain is a segment $W_{1} Z_{1}$ containing $P_{1}$. There exist a $G$-arc $\bar{g}$ and two segments $\bar{W}_{1} \bar{Z}_{1}$ and $\bar{W}_{2} \bar{Z}_{2}$ such that (1) $\bar{W}_{1} \bar{Z}_{1}$ is the set of all points common to $e_{n}$ and $\bar{g}$, (2) $\bar{W}_{2} \bar{Z}_{2}$ is the set of all points common to $e$ and $\bar{g}$, (3) $\bar{W}_{1} \bar{Z}_{1}$ and $\bar{W}_{2} \bar{Z}_{2}$ have a segment in common. Let $\bar{e}_{n}$ denote that part of $e_{n}$ which lies between $\bar{g}$ and the arc of $G$ that contains the upper base of $e_{n}$. Let $\bar{e}_{n+1}$ denote that part of $e$ which lies between $\bar{g}$ and $W_{2} Z_{2}$. The set of elements $e_{1}, e_{2}, e_{3}, \cdots, e_{n-1}, \dot{e}_{n}, \bar{e}_{n+1}$ is a chain of $K$-elements of rank $\epsilon$ from $E$ to $P$. It is thus established that if one point of $W^{\prime} Z^{\prime}$ belongs to $\bar{K}$ then so does every other point of $W^{\prime} Z^{\prime}$. It has been shown that if a $G$-arc above $g_{F}$ contains a point of $\bar{K}$ then so must somé lower arc of $G$. It follows that if $F$ does not belong to $\bar{K}$ there exists an arc $X Y$ which is the uppermost arc of $G$ that contains no point of $\bar{K}$. Let $P$ denote a point of $K$ on the $\operatorname{arc} X Y$. There exists a $K$-element $e$ of rank $\epsilon$ whose lower base contains $P$. The set $G$ contains an $\operatorname{arc} g$ that intersects $e$ in a segment $M N$. 
Let $\bar{P}$ denote a point of $M N$. There exists a chain of $K$-elements of rank $\epsilon$ from $E$ to $\bar{P}$. If to this chain of elements there is added that portion of the $K$-element $e$ which lies between $g$ and $X Y$ there is obtained a chain of $K$ elements of rank $\epsilon$ from $E$ to $P$. Thus the supposition that $E$ can not be joined to $F$ by a chain of $K$-elements of rank $\epsilon$ leads to a contradiction. It follows that there exists a simple chain $e_{11}, e_{12}, e_{13}, \cdots, E_{1 n}$ of $K$-elements of rank 1 from $E$ to $F$. Let $K_{1}$ denote the domain associated with this chain. There exists a simple chain of $K_{1}$-elements of rank 1 from $E$ to $F$. This process may be continued. It follows that there exists a sequence of simple chains $C_{1}, C_{2}, C_{3}, \cdots$ from $E$ to $F$ such that if, for each $n, K_{n}$ denotes the domain associated with $C_{n}$ then (1) every link of $C_{n+1}$ is a $K_{n}$-element of rank $1 / n$, (2) $K_{n+1}^{\prime}$ is a subset of the point-set composed of $K_{n}$ plus its bases. Let $t$ denote the set of all points [ $X$ ] such that $X$ belongs to every $K_{n}$. With the aid of the fact that the set $G$ is equicontinuous, it can be proved* that $t$ is a simple continuous arc from $E$ to $F$ and that it does not have more than one point in common with any given arc of the set $G$. The truth of Lemma 1 is thus established.

Proof of Theorem 1. If $X$ is a point of $A B$ and $X Y$ is that arc of $G$ which has $X$ as one of its endpoints, it may be easily proved with the aid of the Heine-Borel Theorem that there exists

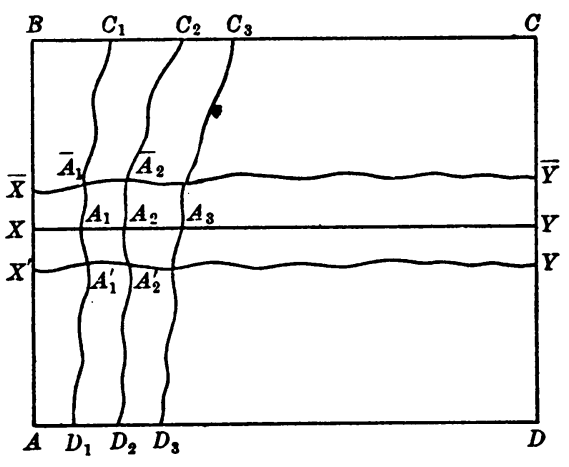

Fig. 4. on $X Y$ a finite set of points $A_{1}, A_{2}$, $A_{3}, \cdots, A_{n}$ in the order $X A_{1} A_{2} A_{3} A_{4} \cdots A_{n-1} A_{n} Y$ such that each of the intervals $X A_{1}, A_{1} A_{2}, \cdots, A_{n-1} A_{n}, A_{n} Y$ of the arc $X Y$ lies wholly within some circle of radius 1 . Let $C_{1}, C_{2}, C_{3}, \cdots, C_{n}$ denote $n$ points in the order $B C_{1} C_{2} C_{3} \cdots C_{n-1} C_{n} C$ on the arc $B C$ and let $D_{1}, D_{2}, D_{3}, \cdots, D_{n}$ denote $n$ points in the order $A D_{1} D_{2} \cdots D_{n-1} D_{n} D$ on the arc $A D$. With the use of Lemma 1 it is easily established that there exist (Fig. 4) two sets of arcs $A_{1} C_{1}, A_{2} C_{2}, A_{3} C_{3}, \cdots, A_{n} C_{n}$ and $A_{1} D_{1}, A_{2} D_{2}, A_{3} D_{3}, \cdots, A_{n} D_{n}$ such that no arc of either set has a point in common with any other arc of that set and such that, for every $n$, (1) $A_{n} C_{n}$ lies except for its endpoints entirely within $A B C D$ and between $X Y$ and $B C$, (2) $A_{n} D_{n}$ lies, except for its endpoints, entirely within $A B C D$ and between $X Y$ and $A D$, (3) neither $A_{n} C_{n}$ nor $A_{n} D_{n}$ has more than one point in common with any one arc of the set $G$. It is easy to show that there exist two points $X^{\prime}$ and $\bar{X}$ in the order $A X^{\prime} X \bar{X} B$

* Cf. the proof of Theorem 15 of my paper On the foundations of plane analysis situs, these Trans a c tions, vol. 17 (1916), pp. 136-139. 
and two $\operatorname{arcs} X^{\prime} Y^{\prime}$ and $\bar{X} \bar{Y}$ belonging to $G$ such that if for every $i(1 \leqq i \leqq n)$ $A_{i}^{\prime}$ is the point in which $X^{\prime} Y^{\prime}$ intersects $A_{i} D_{i}$ and $\bar{A}_{i}$ is the point in which $\bar{X} \bar{Y}$ intersects $A_{i} C_{i}$ then the closed curve bounded by the intervals $A_{i}^{\prime} A_{i}$, $A_{i} \bar{A}_{i}, \bar{A}_{i} \bar{A}_{i+1}, \bar{A}_{i+1} A_{i+1}, A_{i+1} A_{i+1}^{\prime}$ and $A_{i+1}^{\prime} A_{i}^{\prime}$ of the arcs $A_{i} D_{i}, A_{i} C_{i}$, $\bar{X} \bar{Y}, A_{i+1} C_{i+1}, A_{i+1} D_{i+1}$ and $X^{\prime} Y^{\prime}$ respectively (Fig. 4) lies entirely within some circle of radius 1 . For each point $X$ of $A B$ make a similar construction and apply the Heine-Borel Theorem to the set of segments $\left[X^{\prime} \bar{X}\right]$. If certain arcs are properly continued there will result a double ruling* $T_{1}$ of $A B C D$ such that (1) the arcs of one of its single rulings are arcs of $G$ and each arc of its other single ruling has its endpoints on $B C$ and $A D$ respectively and has just one point in common with each arc of the set $G$, (2) each of the subdivisions into which $T_{1}$ divides $A B C D$ lies within some circle of radius 1 . In a similar way each subdivision $\alpha$ of this set can itself be subdivided by a double ruling $T_{1 a}$ such that (1) each arc of one of its single rulings is an interval of an arc of $G,(2)$ each arc of its other single ruling has its endpoints on the arcs which form respectively the upper and the lower base of $\alpha$ and no arc of this ruling has more than one point in common with any arc of $G$, (3) each of the subdivisions inte which $T_{1 a}$ divides $\alpha$ is within a circle of radius $1 / 2$. It follows that there exists a double ruling $T_{2}$ satisfying the Conditions (1) and (2) stated above as being satisfied by $T_{1}$ and also satisfying the additional condition that each of its subdivisions is within some circle of radius $1 / 2$, for every $\alpha$ each arc of $T_{1 a}$ being an interval of an arc of one or the other of the rulings of $T_{2}$. This may be continued. It follows that there exists an infinite sequence of double rulings $T_{1}, T_{2}, T_{3}, \cdots$ such that for every $n$, (1) $T_{n}$ satisfies the conditions (1) and (2) stated above for $T_{1}$, (2) each arc of $T_{n}$ is an arc of $T_{n+1}$, (3) each subdivision of $T_{n}$ is within a circle of radius $1 / n$. Let $\beta$ be the set of all arcs $[t]$ such that, for some $n, t$ belongs to one of the rulings of $T_{n}$ and has its endpoints on $A D$ and $B C$ respectively. If $P$ is a point on $B C$ which is not an endpoint of an arc of the set $\beta$ then there exists just one arc $t_{P}$ that has one endpoint at $P$ and the other on $A D$, lies except for its endpoints entirely within $A B C D$ and has no point in common with any $\operatorname{arc}$ of the set $\beta$. Let $\gamma$ be the set of all such arcs $t_{P}$ for all such points $P$. Let $G^{\prime}$ denote the set of arcs composed of all the arcs of $\beta$ together with all the arcs of $\gamma$ and the straight intervals $A B$ and $C D$. If $P$ is a point on or within the rectangle $A B C D$ let $h_{P}$ denote the distance from $A$ to the point of intersection of $A D$ with that arc of $G^{\prime}$ that passes through $P$. Let $k_{P}$ denote the distance from $A$ to the point in which $A B$ intersects that arc of $G$ which passes through $P$. Let $A D$ be the axis of $X$ and $A B$ the axis of $Y$ in a rectangular system of coördinates. If $P$ is on or within the rectangle $A B C D$ let

* Cf. my paper Concerning a set of postulates for plane analysis situs, these T r a n a a ct i o s , vol. 20 (1919), p. 172 (footnote) and pp. 172-175. 
$P^{\prime}$ denote the point whose coördinates are $\left(h_{P}, k_{P}\right)$. Let $\bar{T}$ denote the transformation of $\bar{R}$ into itself such that if $P$ is any point of $\bar{R}$ then $\bar{T}(P)=P^{\prime}$. It is easy to see that the transformation $\bar{T}$ is continuous and that there exists a continuous transformation $T$, of $S$ into itself, which seduces to $\bar{T}$ on $\bar{R}$. The transformation $T$ satisfies all the requirements of Thəorem 1.

Theorem 2. If, in a plane $S, G$ is a set of open curves such that through each point of $S$ there is just one curve of $G$, then in order that the set of curves $G$ should be in one to one continuous correspondence with a complete system of parallel lines in $S$ it is necessary and sufficient that the set $G$ should be both equicontinunus and inversely equicontinuous with respect to every bounded set of points.

That this condition is necessary may be easily seen. I will show that it is sufficient.

Proof. Suppose that $G$ is a set of open curves such that (1) through each point of $S$ there is just one curve of $G$, (2) $G$ is both equicontinuous and inversely equicontinuous with respect to every bounded set of points. I will first show that of any three distinct curves of the set $G$ one separates the other two from each other.

Suppose on the contrary that there exist three open curves $h, k$ and $l$ of the set $G$ such that no one of them separates the other two. Then the set of all points $[P$ ] such that $P$ is between every two of the curves $h, k$ and $l$ is a domain $D$. Every curve of $G$ which contains a point of $D$ lies wholly in $D$. If $g$ is any curve of $G$ lying wholly in $D$ then either (1) $g$ separates one of the curves $h, k$ and $l$ from the other two or (2) two of the curves $h, k$ and $l$ are such that if they be designated as $\bar{h}$ and $\vec{k}$ respectively and the third one be designated as $\bar{l}$ then there exists a ray $A B$ of $\bar{h}$, a ray $C D$ of $\vec{k}$ and an arc $A C$ lying except for its endpoints wholly in $D$ such that the rays $A B$ and $C D$ and the arc $A C$ constitute the common boundary of a domain $E$ which contains $g$ and is a subset of $D$. A curve $g$ satisfying condition (1) will be called a curve of class I with respect to that one of the curves $h, k$ and $l$ which it separates from the other two, and a curve satisfying condition (2) will be called a curve of class II with respect to $\bar{h}$ and $\bar{k}$. Suppose there exist curves of class I with respect to $h$. It is clear that of every two such curves one of them separates the other one from $h$ and is separated by the other one from $k$ and from $l$. I will show that there is a last curve of class I with respect to $h$, that is to say there is one that separates every other one from $k$ and from $l$. Suppose this is not the case. Let. $K$ denote a point of $k$ and $L$ a point of $l$ and let $K I$ denote an arc which lies except for its endpoints entirely in $D$. In view of the fact that the set of curves $G$ is inversely equicontinuous with respect to the bounded point-set $K L$, it is clear that there exist two other points $K^{\prime}$ and $L^{\prime}$ on $k$ and $l$ respectively and an $\operatorname{arc} K^{\prime} L^{\prime}$ lying, except for its endpoints, 
wholly in $D$ and having no point in common with $K L$ such that (1) the rays $K^{\prime} K$ and $L^{\prime} L$ of $k$ and $l$ respectively, together with the arc $K^{\prime} L^{\prime}$ and the curve $h$, constitute the complete boundary of a domain $\alpha$ which is a subset of $D$ and (2) no arc of $G$ with endpoints on $K L$ contains a point of $K^{\prime} L^{\prime}$. There must exist a point-set $\beta$ (Fig. 5) which is a subset of $\alpha+\operatorname{ray} K^{\prime} K$

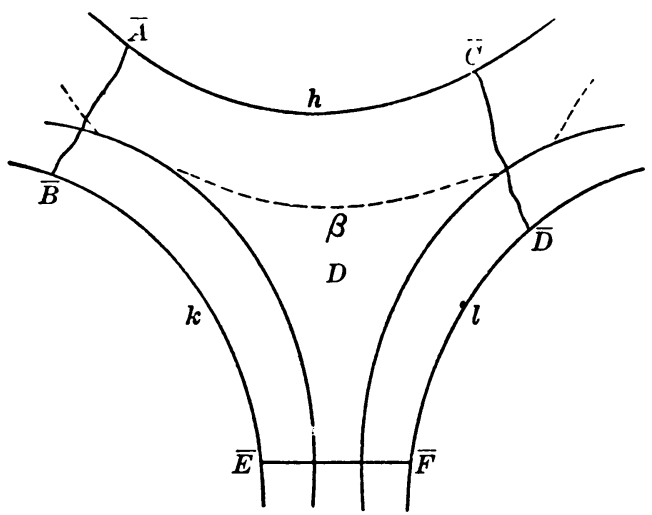

Fig. 5.

+ ray $L^{\prime} L$ such that $\beta$ is the complete boundary of the set of all points [ $X$ ] such that $X$ is separated from $k$ and from $l$ by some curve of class I with respect to $h$. The point-set $\beta$ is connected and contains points in $\alpha$. For each such point $P$ there exists through $P$ a curve $g_{P}$ of the set $G$. Let $\bar{g}_{P}$ denote the set of all those points that are common to $g_{P}$ and $\beta$. The pointset $\bar{g}_{P}$ is a closed proper subset of the connected point-set $\beta$ and has no point in common with $k$ or with $l$. It follows that if $\vec{P}$ is a definite point of $\beta$ lying in $\alpha$ the point-set $\bar{g}_{\bar{P}}$ contains a point $P_{0}$ which is the sequential limit point of a sequence of points $P_{1}, P_{2}, P_{3}, \cdots$, all belonging to $\beta$ and lying in $\alpha$ such that (1) no two of the point-sets $\bar{g}_{P_{1}}, \bar{g}_{P_{2}}, \bar{g}_{P_{3}}, \ldots$ lie on the same curve of the set $G$ and (2) the point-set $P_{1}+P_{2}+P_{3}+\cdots$ is within some closed curve $J$ that lies wholly in $D$. There exist six distinct points $\bar{A}, \bar{B}, \bar{C}, \bar{D}$, $\bar{E}$ and $\bar{F}$ and arcs $\bar{A} \bar{B}, \bar{C} \bar{D}, \bar{E} \bar{F}$ such that (1) $\bar{A}$ and $\bar{C}$ are on $h, \bar{B}$ and $\bar{E}$ are on $k$, and $\bar{D}$ and $\bar{F}$ are on $l$, (2) each of the arcs $\bar{A} \bar{B}, \bar{C} \bar{D}$ and $\bar{E} \bar{F}$ lies, except for its endpoints, in the domain $D$ and no two of them have a point in common, (3) the curve $J$ is wholly within the closed curve $\bar{J}$ formed by the arcs $\bar{A} \bar{B}, \bar{E} \bar{F}$ and $\bar{C} \bar{D}$ together with the intervals $\bar{A} \bar{C}, \bar{B} \bar{E}$ and $\bar{D} \bar{F}$ of the curves $h, k$ and $l$ respectively. There does not exist more than one integer $n$ such that the curve $g_{P_{n}}$ separates $k$ from $h$ and from $l$. For suppose there are two such integers $n_{1}$ and $n_{2}$. Then one of the curves $g_{P_{n_{1}}}$ and $g_{P_{n_{2}}}$ separates the other one from $h$ and therefore separates a point of $\beta$ from $h$. But every domain that contains a point of $\beta$ contains a point of some $G$-curve that separates $h$ from $k$ and from $l$. Hence either $g_{P_{n_{1}}}$ or $g_{P_{n_{2}}}$ separates $h$ 
from a $G$-curve $g_{0}$ which separates $h$ from $k$ and from $l$. Hence $g_{P_{n_{2}}}$ or $g_{P_{n_{3}}}$ has at least one point in common with $g_{0}$. But this is contrary to hypothesis. Similarly there does not exist more than one value of $n$ such that $g_{P_{n}}$ separates $l$ from $h$ and from $k$. It follows that there exists an infinite sub-sequence $g_{P_{n_{1}}}, g_{P_{n_{2}}}, g_{P_{n_{3}}}, \cdots$ of distinct curves of the sequence $g_{P_{1},} g_{P_{3}}, g_{P_{3}}, \cdots$ and an arc $X Y$ (identical with one of the arcs $\bar{A} \bar{B}, \bar{C} \bar{D}$ and $\bar{E} \bar{F}$ ) such that for each $m$ the curve $g_{P_{n_{m}}}$ contains an interval $A_{P_{n_{m}}} P_{n_{m}} B_{P_{n_{m}}}$ whose endpoints $A_{P_{n}}$ and $B_{P_{n}}$ are on $X \dot{Y}$ and which lies, except for its endpoints, wholly within $\bar{J}$. By hypothesis, for every positive number $\epsilon$ there exists a positive number $\delta_{J_{e}}$ such that if, for some $n$, the distance from $A_{P_{n_{m}}}$ to $B_{P_{n_{m}}}$ is less than $\delta_{\bar{J}_{\epsilon}}$ then the whole arc $A_{P_{n_{m}}} P_{n_{m}} B_{P_{n_{m}}}$ lies within a circle of radius $\epsilon$. It can be easily seen that if $i$ and $j$ are distinct integers the intervals $A_{P_{n_{i}}} B_{P_{n_{i}}}$ and $A_{P_{n},} B_{P_{n},}$ of the arc $X Y$ have no point in common. Hence if $\epsilon$ is the least distance from a point of the arc $X Y$ to a point of the closed curve $J$ there exists an integer $\bar{m}$ such that the distance from $A_{P_{n_{\bar{m}}}}$ to $B_{P_{n_{\bar{m}}}}$ is less than $\delta_{\vec{J}_{e}}$. It follows that every point of the arc $A_{P_{n_{\bar{m}}}} P_{n_{\bar{m}}} B_{P_{n_{\bar{n}}}}$ is at a distance of less than $\epsilon$ from the point $A_{P_{n_{\bar{m}}}}$. But the distance from $P_{n_{\bar{m}}}$ to $A_{P_{n_{\bar{m}}}}$ is not less than $\epsilon$. Thus the supposition that there exists no last curve of class I with respect to $h$ has led to a contradiction. Hence there exists a curve $\bar{h}$ which is the last curve of class I with respect to $h$. In a similar way it may be shown that there exist curves $\bar{k}$ and $\bar{l}$ which are the last curves of class I with respect to $k$ and $l$ respectively. No one of the curves $\bar{h}, \vec{k}$ and $\vec{l}$ separates the other two from each other and no curve of the set $G$ separates one of them from the other two.

Let $\bar{D}$ denote the connected domain which is bounded by the curves $\bar{h}$, $\bar{k}$ and $\bar{l}$. With the aid of several applications of the fact that the system $G$ is inversely equicontinuous with respect to every bounded set of points it can be shown that there exist six points $\bar{M}, \bar{T}, \bar{H}, \bar{L}, \bar{K}, \bar{N}$ and three ares $\bar{M} \bar{N}$, $\bar{T} \bar{H}$ and $\bar{K} \bar{L}$ such that (1) $\bar{M}$ and $\bar{T}$ are on $\bar{h}, \bar{H}$ and $\bar{L}$ are on $\bar{l}, \bar{K}$ and $\bar{N}$ are on $\bar{k}$, (2) $\bar{M} \bar{N}, \bar{T} \bar{H}$ and $\bar{K} \bar{L}$ lie, except for their endpoints, entirely in $\bar{D}$ and no two of them have a point in common, (3) no curve of $G$ distinct from $\bar{h}$, $\bar{k}$ and $\bar{l}$ contains a point of more than one of the $\operatorname{arcs} \bar{M} \bar{N}, \bar{T} \bar{H}$ and $\bar{K} \bar{L}$ (Fig. 6). Let $\bar{\beta}$ denote the region bounded by the arcs $\bar{M} \bar{N}, \bar{T} \bar{H}, \bar{K} \bar{L}$ and the intervals $\bar{M} \bar{T}, \bar{H} \bar{L}$ and $\bar{K} \bar{N}$ respectively of the curves $\bar{h}, \bar{l}$ and $\bar{k}$. By an argument similar in large part to that employed above to show the existence of $\bar{h}$ it may be proved that if $g$ is a curve of the set $G$ that contains a point of $\bar{M} \bar{N}$ there exists a curve $\bar{g}$ of the set $G$ which either coincides with $g$ or separates $g$ from each of the curves $\bar{h}, \bar{k}$ and $\bar{l}$ but is not itself separated from any one of these curves by any other curve of $G$. Every such curve $\bar{g}$ will be called a curve of class III. There clearly exist infinitely many distinct curves of class III. Let $M^{*}$ denote a point on $\bar{h}$ in the order $\bar{T} \bar{M} \bar{M}^{*}$ and let $N^{*}$ denote a 
point on $\bar{k}$ in the order $\bar{K} \bar{N} N^{*}$. Let $t$ denote an arc that has $M^{*}$ and $N^{*}$ as endpoints, lies except for its endpoints entirely in $\bar{D}$ and has no point in common with $\bar{M} \bar{N}$. If a curve of $G$ contains a point $P$ of $\bar{M} \bar{N}$ there is an interval of that curve that contains $P$ and has its endpoints on $t$. It follows that there exists an infinite set of distinct $\operatorname{arcs} A_{1} P_{1} B_{1}, A_{2} P_{2} B_{2}, A_{3} P_{3} B_{3}, \cdots$ such that (1) for every $n, A_{n}$ and $B_{n}$ are on $t$ and the $\operatorname{arc} A_{n} P_{n} B_{n}$ is an interval of a curve of class III, (2) if $n_{1}$ is distinct from $n_{2}, A_{n_{1}} P_{n_{1}} B_{n_{1}}$ and $A_{n_{2}} P_{n_{2}} B_{n_{2}}$

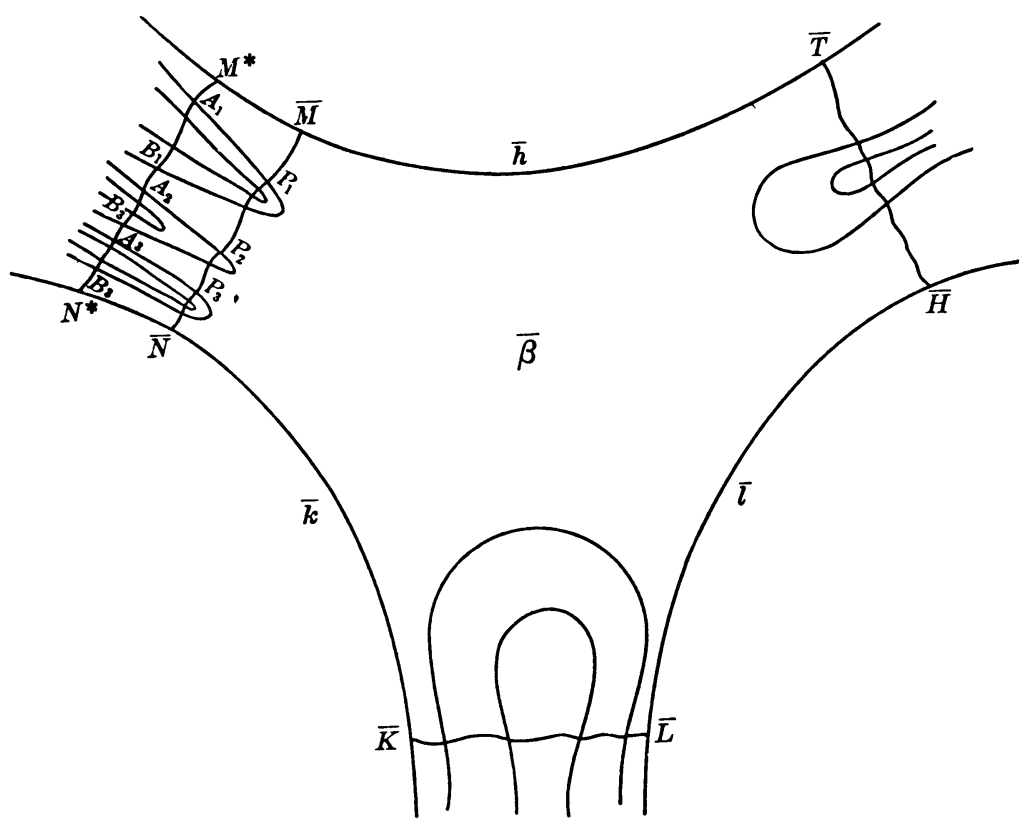

FIG. 6.

are not intervals of the same curve of the set $G$, (3) for every $n$ the arc $A_{n} P_{n} B_{n}$ contains a point of $\bar{M} \bar{N}$, (4) if $n_{1}$ is distinct from $n_{2}$ the intervals $A_{n_{1}} B_{n_{1}}$ and $A_{n_{3}} B_{n_{3}}$ of $M^{*} N^{*}$ have no point in common. If $\epsilon$ is the least distance from a point of $t$ to a point of $\bar{M} \bar{N}$ there exists a positive integer $n$ such that the distance from $A_{\bar{n}}$ to $B_{\bar{n}}$ is less than $\delta_{t \mathrm{e}}$. But there exists an interval $A_{\bar{n}} P_{\bar{n}} B_{\bar{n}}$ of a curve of the set $G$ with $A_{\bar{n}}$ and $B_{\bar{n}}$ as endpoints and containing a point $P_{\bar{n}}$ at a distance of $\epsilon$ or more from $A_{\vec{n}}$. Thus the supposition that no one of the curves $h, k$ and $l$ separates the other two has led to a contradiction. It follows that, of any three curves of $G$, one separates the other two.

If $\bar{g}$ is a definite $G$-curve, $C$ is a definite circle and $A$ is a point of $C$ not lying on $\bar{g}$ there exists a $G$-curve which lies on the $A$-side of $\bar{g}$ but contains

* See Theorem 2 of my paper On the most general class $L$ of Fréchet in which the Heine-BorelLebesgue Theorem holds true, Proceedings of the National Academy of S ci e n c e s, vol. 5 (1919), p. 208. 
no point of $C$. This may be proved as follows. For every $G$-curve $g$ that lies on the $A$-side of $\vec{g}$ and contains a point of $C$ let $C_{o}$ denote the set of all points [X] of $C$ such that $X$ is either on $g$ or on the far side of $g$ from $\bar{g}$. For every such $g$ the set $C_{g}$ is closed and bounded and for every two such $g$ 's, $\bar{g}_{1}$ and $\bar{g}_{2}$, either $C_{\bar{g}_{1}}$ contains $C_{\bar{\theta}_{2}}$ or $C_{\bar{\theta}_{2}}$ contains $C_{\bar{\theta}_{1}}$. It follows* that there exists at least one point $P$ whicn belongs to $C_{g}$ for every $G$-curve $g$ which lies on the $A$-side of $\vec{g}$ and contains a point of $C$. If $g_{P}$ denotes that $G$-curve which contains the point $P$ then no $G$-curve which lies on the far side of $g_{P}$ from $\bar{g}$ can contain any point of $C$.

It easily follows that for every circle $C$ there exist two $G$-curves such that every point of $C$ lies between them. Now let $O$ denote some definite point and for each positive integer $n$ let $C_{n}$ denote a circle with center at $O$ and radius $n$. Let $g_{0}$ denote that $G$-curve which passes through $O$. Let $g_{1}$ and $g_{-1}$ denote two $G$-curves such that $C_{1}$ lies between them. Let $g_{2}$ and $g_{-2}$ denote two $G$-curves such that $C_{2}$ lies between them and such that $g_{2}$ is on the far side of $g_{1}$ from $O$ and $g_{-2}$ is on the far side of $g_{-1}$ from $O$. This process may be continued. It follows that there exists a set $G_{0}$ of $G$-curves consisting of two infinite sequences $g_{0}, g_{1}, g_{2}, \cdots$ and $g_{-1}, g_{-2}, g_{-3}^{-}, \cdots$ such that, for each positive $n, C_{n}$ lies between $g_{n}$ and $g_{-n}, g_{n+1}$ is on the far side of $g_{n}$ from $O$ and $g_{-(n+1)}$ is on the far side of $g_{-n}$ from $O$. It is clear that every point is either on some curve of the set $G_{0}$ or between two successive curves of $G_{0}$. With the use of the fact that the system $G$ is inversely equicontinuous with respect to every bounded point-set and that, of any three curves of $G$, one separates the other two, it can be shown that there exist four infinite sequences of points $A_{0}, A_{1}, A_{2}, A_{3}, \cdots ; A_{-1}, A_{-2}, A_{-3}, \cdots ; B_{0}, B_{1}$, $B_{2}, B_{3}, \cdots$ and $B_{-1}, B_{-2}, B_{-3}, \cdots$ and two sequences of arcs $A_{0} B_{0}$, $A_{1} B_{1}, A_{2} B_{2}, \cdots$ and $A_{-1} B_{-1}, A_{-2} B_{-2}, A_{-3} B_{-3}, \cdots$ such that (1) for every $n$ the points $A_{n}, A_{n+1}, A_{n+2}$ are in the order $A_{n} A_{n+1} A_{n+2}$ on $g_{1}$ and the points $B_{n}, B_{n+1}, B_{n+2}$ are in the order $B_{n} B_{n+1} B_{n+2}$ on $g_{0}$, (2) for each $n$ and $m(m \neq n)$ the $\operatorname{arcs} A_{n} B_{n}$ and $A_{m} B_{m}$ lie entirely between $g_{0}$ and $g_{1}$ and have no point in common, (3) for every point $X$ on $g_{1}$ and every point $Y$ on $g_{0}$ there exists a positive integer $n$ such that $X$ is on the interval $A_{-n} A_{n}$ of $g_{1}$ and $Y$ is on the interval $B_{-n} B_{n}$ of $g_{0}$, (4) if, for each $n, J_{n}$ denotes the closed curve formed by the $\operatorname{arcs} A_{n} B_{n}, A_{n+1} B_{n+1}$ and the $G$-intervals $A_{n} A_{n+1}$ and $B_{n} B_{n+1}$ then (a) every point between $g_{0}$ and $g_{1}$ is on or within some $J_{n}$, (b) if $|m-n|>1$ every $G$-interval whose endpoints are on or within $J_{m}$ lies wholly without $J_{n}$. For each integer $n$ let $K_{n}$ denote the set of all points [ $X$ ] such that $X$ lies on a $G$-interval whose endpoints are within $J_{4 n}$. By methods wholly or largely identical with those employed in the proof of Lemma 1 it may be shown that there exists an arc $D_{n} E_{n}$ which lies entirely in the domain $K_{n}$, except that its endpoints $D_{n}$ and $E_{n}$ lie on $g_{1}$ and $g_{0}$ respectively, 
and which does not have more than one point in common with any curve of the set $G$. For each $n$ let $\bar{J}_{n}$ denote the closed curve found by the arcs $D_{n} E_{n}, D_{n+1} E_{n+1}$ and the $G$-intervals $D_{n} D_{n+1}, E_{n} E_{n+1}$ and let $\bar{R}_{n}$ denote its interior. By Theorem 1 there exists a set of arcs $\alpha_{n}$ such that (1) each arc of $\alpha_{n}$ has its endpoints on $g_{1}$ and $g_{0}$ respectively and lies, except for its endpoints, wholly within $\vec{J}_{n},(2)$ no two arcs of $\alpha_{n}$ have a point in common, (3) through each point of the point-set composed of $\bar{R}_{n}$ and the two $G$-segments $D_{n} D_{n+1}$ and $E_{n} E_{n+1}$ there is one and only one arc of the set $\alpha_{n}$, (4) no arc of $\alpha_{n}$ has more than one point in common with any one arc of the set $G$. Let $H_{0}$ denote the set of arcs composed of all the arcs of all the sets $\alpha_{n}$ together with all the $\operatorname{arcs} D_{n} E_{n}$. For each $n$ there exists a set of $\operatorname{arcs} H_{n}$ bearing to $g_{n}$ and $g_{n+1}$ a relation similar to the above described relation of $H_{0}$ to $g_{0}$ and $g_{1}$, so that (1) each arc of $H_{n}$ lies entirely between $g_{n}$ and $g_{n+1}$ except that its endpoints are on $g_{n}$ and $g_{n+1}$ respectively, (2) through each point that lies on $g_{n}$ or $g_{n+1}$ or between them there is just one arc of $H_{n}$, (3) no arc of $H_{n}$ has more than one point in common with any arc of the set $G$. For each point $P$ there exists $n_{P}$ such that $P$ is either on $g_{n_{P}}$ or between $g_{n_{P}}$ and $g_{n_{P}+1}$. Let $h_{1 P}$ denote that arc of $H_{n_{P}}$ which passes through $P$. Let $h_{2 P}$ denote that arc of $H_{n_{P}+1}$ which has an endpoint in common with $h_{1 P}$ and let $h_{0 P}$ denote that arc of $H_{n_{P}-1}$ which has an endpoint in common with $h_{1 P}$. This process may be continued. Thus there exists a set of arcs $\left[h_{m P}\right](-\infty<m<\infty)$ such that, for every $m, h_{(m+1) P}$ belongs to the set $H_{n_{P}+m}$ and has an endpoint in common with $h_{(m+2) P}$. The point-set obtained by adding together all the arcs of the set $\left[h_{m P}\right]$ is an open curve $h_{P}$ that passes through the point $P$ and has just one point in common with each curve of the set $G$. Let $H$ denote the set of all curves $h_{P}$ for all points $P$ of $S$. Through each point of $S$ there is just one curve.of the set $H$ and just one curve of the set $G$ and if $h$ is any curve of $H$ and $g$ is any curve of $G, h$ and $g$ have just one point in common. It follows* that there exists a one to one transformation of $S$ into itself which carries $H$ into a complete system of parallel lines and $G$ into another complete system of parallel lines.

Theorem 3. If $A \bar{A}_{0} \bar{B}_{0} B$ is a rectangle and $A_{1} B_{1}, A_{2} B_{2}, A_{3} B_{3}, \cdots$ is an infinite sequence $G$ of arcs such that (1) the points $A_{1}, A_{2}, A_{3}, \cdots$ are in the order $\bar{A}_{0} A_{1} A_{2} A_{3} \cdots A_{n} A_{n+1} \cdots A$ on the interval $\bar{A}_{0} A$ and the points $B_{1}, B_{2}, B_{3}, \cdots$ are in the order $\bar{B}_{0} B_{1} B_{2} B_{3} \cdots B_{n} B_{n+1} \cdots B$ on the interval $\bar{B}_{0} B,(2)$ every arc of $G$ lies, except for its endpoints entirely within the rectangle $A \bar{A}_{0} \bar{B}_{0} B$, (3) no two arce of $G$ have a point in common and (4) for each positive number $\epsilon$ there exists a positive number $n_{\text {s }}$ such that if $n>n_{e}$, then every point of $A_{n} B_{n}$ is at a distance less than $\epsilon$ from the line $A B$; then in order that the sequence $G$

* Cf. pp. 177-178 of my paper Concerning a set of postulates for plane analysis situs, loc. cit. 
should be equivalent from the standpoint of analysis situs to an infinite sequence of straight line intervals, satisfying the same conditions (1)-(4), and all parallel to $A B$, it is necessary and sufficient that the set of arcs $G$ should be equicontinuous.

That this condition is necessary, is evident. I will show that it is sufficient.

Proof. Suppose $G$ is an equicontinuous sequence of arcs satisfying conditions (1)-(4) of the hypothesis of Theorem 3. By hypothesis for every positive number $\epsilon$ there exists a positive number $\delta_{e}$ such that if $P_{1}$ and $P_{2}$ are two points on an arc $g$ of $G$ at a distance apart less than or equal to $\delta_{e}$ then the interval $P_{1} P_{2}$ of $g$ lies entirely within some circle of radius $\epsilon$. It follows with the help of condition (4) that if $X$ and $Y$ are two points of $A B$ at a distance apart less than or equal to $\delta_{\mathrm{e}}$ and $p_{1}$ and $p_{2}$ are straight lines perpendicular to $A B$ at $X$ and $Y$ respectively then if* $n>n_{\delta_{e}}$ no interval of $A_{n} B_{n}$ with endpoints on $p_{1}$ contains a point of $p_{2}$. If, for every $n, X_{n}$ denotes the last point that $A_{n} B_{n}$ has in common with $p_{1}$ and $Y_{n}$ denotes the first point that it has in common with $p_{2}$ it follows that if $n_{1}$ and $n_{2}$ are positive integers greater than $n_{\delta_{e}}$ and $P_{n_{1}}$ and $P_{n_{2}}$ are points between $p_{1}$ and $p_{2}$ on the intervals $X_{n_{1}} Y_{n_{1}}$ and $X_{n_{2}} Y_{n_{2}}$ respectively of the arcs $A_{n_{1}} B_{n_{1}}, A_{n_{2}} B_{n_{2}}$ then $P_{n_{1}}$ can be joined to $P_{n_{2}}$ by a simple continuous arc that lies wholly between $p_{1}$ and $p_{2}$ and lies except for its endpoints wholly between the $\operatorname{arcs} A_{n_{1}} B_{n_{1}}$ and $A_{n_{2}} B_{n_{2}}$. Now for each positive integer $n$ subdivide the interval $A B$ into $3^{n}$ equal subintervals by $3^{n}-1$ points $A_{n 1}, A_{n 2}, A_{n 3}, \cdots, A_{n\left(3^{n}-1\right)}(1 \leqq n<\infty$.) in the order $A A_{n_{1}} A_{n_{3}} \cdots A_{n\left(3^{n}-1\right)} B$. For each $n$ and $m\left(1 \leqq m \leqq 3^{n}-1\right)$ let $p_{n m}$ denote the perpendicular to $A B$ at the point $A_{n m}$. There exists a sequence of positive integers $\bar{n}_{1}, \bar{n}_{2}, \bar{n}_{3}, \cdots$ such that $\bar{n}_{1}<\bar{n}_{2}<\bar{n}_{3} \cdots$ and such that, for every $k, \bar{n}_{k}>n_{\delta_{l / 3 k}}$, where $l$ is the length of $A B$. For each $k$ let $\bar{g}_{k}$ denote the arc $A_{\bar{n}_{k}} B_{\bar{n}_{k}}$ and let $\bar{A}_{k}$ and $\bar{B}_{k}$ denote its endpoints, $\bar{A}_{k}$ being that one which lies on $A \bar{A}_{0}$. For each $n$ and $m\left(1 \leqq m \leqq 3^{n}-1\right)$ let $B_{n m}$ be the airst and $A_{n m}$ the last point that the arc $\bar{g}_{n}$ has in common with $p_{n m}$. Let $t_{n m}$ denote the $G$-segment $A_{n m} B_{n(m+1)}$. For each $n$ and each positive integer $\dot{m}$ (less $\operatorname{than} 3^{n}$ ) of the form $3 k-2$ (where $k$ is an integer) let $X_{n m}$ denote a point of the segment $t_{n m}$. If, for each such $n$ and $m, \bar{m}$ denotes the number $3 m+1$, there exists (Fig. 7) an arc $X_{n m} X_{(n+1) \bar{m}}$ which has not† more than one point in common with any arc of the set $G$, lies wholly between the lines $p_{n m}$ and $p_{n(m+1)}$ and also lies, except for its endpoints, wholly between the arcs $\bar{g}_{n}$ and $\bar{g}_{n+1}$. For every $n(0 \leqq n<\infty)$ let $A_{n}^{\prime}$ denote a point on the straight line interval $\bar{A}_{0} A$ at a distance from $A$ equal to $a /(n+1)$, where $a$ is the length of $\bar{A}_{0} A$, and let $B_{n}^{\prime}$ denote a point on the interval $\bar{B}_{0} B$ at the distance $a /(n+1)$ from $B$. Let $\bar{g}_{0}$ denote the straight line interval $\bar{A}_{0} \bar{B}_{0}$. For each $n(0 \leqq n<\infty)$ let $J_{n}$ denote the closed curve formed by the arcs $\bar{g}_{n}$ and $\bar{g}_{n+1}$ and the intervals

${ }^{*}$ For the meaning of $n_{\delta_{\mathrm{e}}}$ see Condition (4).

$\dagger$ There are not more than a finite number of arcs. of the set $G$ between $\bar{g}_{n}$ and $\bar{g}_{n+1}$. 
$\bar{A}_{n} \bar{A}_{n+1}$ and $\bar{B}_{n} \bar{B}_{n+1}$ of $A \bar{A}_{0}$ and $B \bar{B}_{0}$ respectively. Let $R_{n}$ denote the pointset composed of $J_{n}$ and its interior. Let $R_{n}^{\prime}$ denote the point-set composed of the rectangle $A_{n}^{\prime} A_{n+1}^{\prime} B_{n+1}^{\prime} B_{n}^{\prime}$ and its interior and let $R$ be that composed of $A \bar{A}_{0} \bar{B}_{0} B$ and its interior. Let $b$ denote the length of $A B$. With the aid of a theorem of Schoenflies'* it may be easily seen that there exists a sequence of one to one transformations $T_{0}, T_{1}, T_{2}, \cdots$ such that, for each $n$ ( $0 \leqq n$ $<\infty)$, (1) $T_{n}$ is a continuous transformation of $R_{n}$ into $R_{n}^{\prime}$, (2) $T_{n}$ trans-

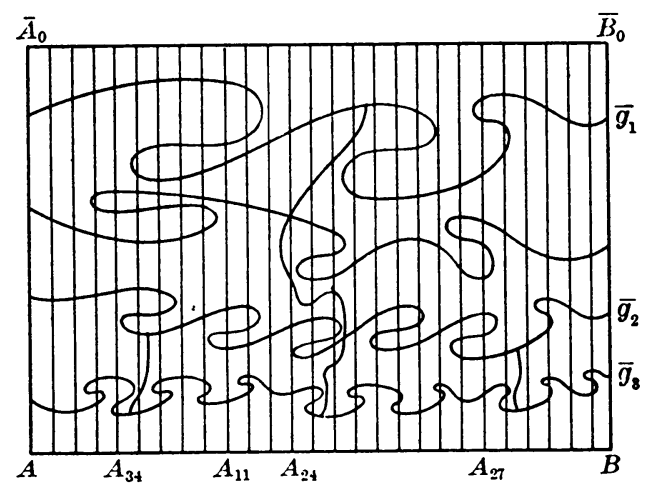

FIG. 7.

forms $\bar{A}_{n}, \bar{A}_{n+1}, \bar{B}_{n}$ and $\bar{B}_{n+1}$ into $A_{n}^{\prime}, A_{n+1}^{\prime}, B_{n}^{\prime}$ and $B_{n+1}^{\prime}$ respectively, (3) if $P$ is a point of $\bar{g}_{n+1}, T_{n}(P)=T_{n+1}(P)$, (4) if there is any $G$-arc between $\bar{g}_{n}$ and $\bar{g}_{n+1}$ every such arc is transformed by $T_{n}$ into a straight line interval parallel to $A B$, (5) if $n \geqq 1$ then for each $m$ (less than $3^{n}$ ) of the form $3 k-2$, where $k$ is a positive integer, the point $X_{n m}$ is transformed by $T_{n}$ into a point $X_{n m}^{\prime}$ lying on the straight interval $A_{n}^{\prime} B_{n}^{\prime}$ at a distance from $A \bar{A}_{0}$ equal to $(m+1 / 2) b / 3^{n}$ and the arc $X_{n m} X_{(n+1) \bar{m}}$ is transformed into the straight line interval joining the point $X_{n m}^{\prime}$ to the point $X_{(n+1) \bar{m}}^{\prime}$. For each $n$ let $H_{n}$ denote the set of all arcs $[h]$ in $R_{n}$ such that $T_{n}(h)$ is a vertical $\dagger$ straight line interval. If $\bar{P}$ is a point on $\bar{A}_{0} \bar{B}_{0}$ let $h_{\bar{P} 0}$ denote that arc of $H_{0}$ which contains $\bar{P}$, let $h_{\bar{P} 1}$ denote that arc of $H_{1}$ which has an endpoint in common with $h_{\bar{P} 0}$, let $h_{\bar{P} 2}$ denote that arc of $H_{2}$ which has an endpoint in common with $h_{\bar{P} 1}$, and so on indefinitely. It is possible to show that there exists only one point $O_{\bar{P}}$ on $A B$ which is a limit point of the point-set $h_{\bar{P} 0}+h_{\bar{P} 1}+h_{\bar{P} 2}+\cdots$ and that the set of points $O_{\bar{P}}+h_{\bar{P} 0}+h_{\bar{P} 1}+h_{\bar{P} 2}+\cdots$ is a simple continuous arc from $\bar{P}$ to $O_{\bar{P}}$. Let $H$ denote the set of all such arcs for all points $\bar{P}$ on $\bar{A}_{0} \bar{B}_{0}$. Let $K$ denote the set of arcs composed of $A B$ and every arc in $R$ which, for some $n$, is transformed by $T_{n}$ into a straight interval parallel to $A B$. If $P$ is any point of $R$ let $O_{P}$ denote the point which $A B$ has in common with

* Bericht über die Entwickelung der Lehre von den Punktmannigfaltigkeiten, Part II, p. 108. $\dagger$ I.e., perpendicular to $A B$. 
that arc of $H$ which passes through $P$ and let $L_{P}$ denote the point which $\bar{A}_{0} A$ has in common with that arc of $K$ which passes through $P$. For each point $P$, of $R$, let $T(P)$ denote the point in which the perpendicular to $A B$ at the point $O_{P}$ intersects the perpendicular to $A \bar{A}_{0}$ at the point $L_{P}$. The so determined transformation $T$ is a continuous transformation of $R$ into itself. It is easy to see that there exists a continuous transformation of $S$ into itself which reduces to $T$ on $R$. Every such transformation satisfies the requirements of Theorem 3.

The truth of the following theorems may also be established.

Theorem 4. If, in a plane $S, O$ is a point and $G$ is a set of open curves through $O$ such that through each point of $S$ distinct from $O$ there is one and only one curve of the set $G$, then in order that $G$ should be equivalent from the standpoint of analysis situs to the set of all straight lines in $S$ through $O$ it is necessary and sufficient that $G$ should be equicontinuous with respect to every bounded set of points.

Theorem 5. If, in a plane $S, O$ is a point and $G$ is a set of simple closed curves enclosing $O$ such that through each point of $S$ distinct from $O$ there is one and only one curve of the set $G$, then in order that $G$ should be equivalent from the standpoint of analysis situs to the set of all circles in $S$ with center at $O$ it is necessary and sufficient that the set $G$ should be equicontinuous with respect to every bounded set of points.

University of TeXas 